\title{
ENHANCEMENT OF NON-INVASIVE TRANS-MEMBRANE DRUG DELIVERY USING ULTRASOUND AND MICROBUBBLES DURING PHYSIOLOGICALLY RELEVANT FLOW
}

FARAH E. SHAMOUT, ANTONIOS N. POULIOPOULOS, PATRIZIA LEE, SIMONE BONACCORSI, LEILA TOWHIDI, ROB KRAMS, JAMES J. CHOI

10

Department of Bioengineering, Imperial College London, London, United Kingdom

Address correspondence to: James J. Choi, Noninvasive Surgery and Biopsy Laboratory, Bioengineering Department, Imperial College London, London, SW7 2AZ, United 20 Kingdom.

E-mail: j.choi@imperial.ac.uk 


\begin{abstract}
\end{abstract}
Sonoporation has been shown able to deliver drugs across cell membranes and into target cells, yet several limitations have prohibited further advancement of this technology.

5 Higher delivery rates were associated with increased cellular death, thus implying a safety-efficacy trade-off. Meanwhile, there has been no reported study of safe in vitro sonoporation in a physiologically relevant flow environment. The objective of our study was not only to evaluate sonoporation under physiologically relevant flow conditions, such as fluid velocity, shear stress, and temperature, but also to design ultrasound

10 parameters that exploit the presence of flow to maximize sonoporation efficacy while minimizing or avoiding cellular damage. Human umbilical vein endothelial cells (EA.hy926) were seeded in flow chambers as a monolayer to mimic the endothelium. A peristaltic pump maintained a constant fluid velocity of $12.5 \mathrm{~cm} / \mathrm{s}$. A focused $0.5 \mathrm{MHz}$ transducer was used to sonicate the cells, while an inserted focused $7.5 \mathrm{MHz}$ passive

15 cavitation detector monitored microbubble-seeded cavitation emissions. Under these conditions, propidium iodide, which is normally impermeable to the cell membrane, was traced to determine whether it could enter cells after sonication. Meanwhile, calcein-AM was used as a cell viability marker. A range of focused ultrasound parameters was explored, with several unique bioeffects observed: cell detachment, preservation of cell

20 viability with no membrane penetration, cell death, and preservation of cell viability with sonoporation. The parameters were then modified further to produce safe sonoporation with minimal cell death. To increase the number of favourable cavitation events, we lowered the ultrasound exposure pressure to $40 \mathrm{kPa}_{\mathrm{pk}-\mathrm{neg}}$ and increased the number of 
cavitation nuclei by 50 times, to produce a trans-membrane delivery rate of $62.6 \pm 4.3 \%$ with a cell viability of $95 \% \pm 4.2 \%$. Furthermore, acoustic cavitation analysis showed that the low pressure sonication produced stable and non-inertial cavitation throughout the pulse sequence. To our knowledge, this is the first study to demonstrate a high drug 5 delivery rate coupled with high cell viability in a physiologically relevant in vitro flow system.

Key Words: microbubbles; ultrasound; trans-membrane drug delivery; sonoporation; endothelial cells; EA.hy926 


\section{INTRODUCTION}

Focused ultrasound (FUS) techniques are being developed to drive acoustic cavitation - acoustically driven microbubble activity - and produce therapeutic biological

5 effects at specified locations (Dalecki, 2004; Stride and Coussios, 2010). Amongst a multitude of methods, trans-membrane molecular delivery has been of great interest due to its potential to address one of the most pressing challenges with pharmaceuticals: drug transport into cells. The application of FUS and microbubbles facilitates trans-membrane transport by generating acoustic cavitation at or near the cell membrane, but the exact

10 physical and biological mechanisms involved remain unknown (Schlicher et al., 2006; Zeghimi et al., 2012). Proposed routes of molecular delivery include the formation of short-lived pores in the membrane, also known as sonoporation ( $\mathrm{Yu}$ and $\mathrm{Xu}, 2014)$, and endocytosis (Geers et al., 2011; van Wamel et al., 2006). In addition, increased transmembrane delivery efficiency is associated with increased damage and cell death (Qiu et

15 al., 2012, 2010), and this efficacy-safety trade-off has limited the technology's development and hence it remains unutilized in the clinic as a drug delivery tool.

Ultrasonic trans-membrane delivery requires microbubbles undergoing acoustic cavitation to act near or on the surface of the cell membrane (Ohl et al., 2006; Prentice et al., 2005; Zhou et al., 2012). Ultrasound is typically generated from a focused transducer

20 outside the body and can propagate several centimeters into the body to converge to a small focal volume. Microbubbles, which are typically $1-10 \mu \mathrm{m}$ in diameter, coated with a protein, polymer, or lipid shell, and filled with a stabilized gas core, are then systemically administered so that they circulate the body's diverse vasculature. 
Microbubbles are clinically approved and being used as contrast agents in ultrasound imaging (Cosgrove, 2006). However, their use is extended to therapeutic applications such as sonoporation and targeted drug delivery applications (Ferrara et al., 2007). Microbubbles can flow at speeds varying from $20 \mathrm{~cm} / \mathrm{s}$ in the middle of the vessel to a

5 few $\mathrm{mm} / \mathrm{s}$ near the vessel wall, which correlate well with the velocity of red blood cells (Levine et al., 1984). When exposed to ultrasound, microbubbles respond in a complex range of behaviors known as acoustic cavitation (Apfel, 1997). Ultrasound exposure parameters, such as center frequency, peak-rarefactional pressure, pulse length, pulse repetition frequency, etc. can be adjusted to control the cavitation magnitude, duration

10 and distribution (Choi and Coussios, 2012; Dalecki, 2004).

Depending on the ultrasound parameters employed, the physical composition of the microbubbles under ultrasound exposure, and the environment surrounding the microbubble, different types of cavitation can occur. Microbubbles undergoing acoustic cavitation are expanding and contracting in response to the compressional and

15 rarefactional phases of the ultrasound wave (Apfel, 1997; Helfield and Goertz, 2013). At low acoustic pressures, microbubbles recurrently oscillate around an equilibrium radius in a behavior known as stable cavitation (Apfel, 1997; Dalecki, 2004; Morgan et al., 2000). Transient cavitation describes short-lived phenomena, which can be caused by fragmentation of the shell, dissolution of the core gas, or other mechanisms (Apfel, 1997;

20 Chomas et al., 2001; Newman and Bettinger, 2007). One cause of a transient response is inertial cavitation, which arises when the rarefactional phase of the ultrasound pulse results in a radial expansion followed by a violent collapse dominated by the inertia of the surrounding liquid medium (Church and Carstensen, 2001; Flynn, 1982). 
The different types of acoustic cavitation have been demonstrated to produce diverse cellular effects (Fan et al., 2014; Mehier-Humbert et al., 2005; Qiu et al., 2010). First, it has been shown that the interaction between the microbubble oscillation and the cell membrane can produce short-lived pores that allow drug uptake. These pores are up

5 to $1 \mu \mathrm{m}$ in size and have been shown to reseal within seconds (Hu et al., 2013; Newman and Bettinger, 2007; Qiu et al., 2010; Zhou et al., 2009), minutes (Schlicher et al., 2006) or even hours (Zhao et al., 2008). Inertial cavitation produces higher rates of intracellular delivery than stable cavitation (Fan et al., 2013; Karshafian et al., 2009; Park et al., 2011; Qiu et al., 2010), however the cellular membrane is more likely to be disrupted and that

10 influences cell viability (Newman and Bettinger, 2007). Furthermore, at diagnostic frequencies and acoustic pressures, endothelial damage can occur when contrast agents are under ultrasound exposure, due to the generation of inertial cavitation. Such cavitation resulted in severe cell erosion from in vitro static monolayers (Brayman et al., 1999) and in cell lysis (Ward et al., 1999). Thus, non-inertial cavitation is deemed safer in

15 comparison to inertial cavitation, due to its reduced effect on cell viability (Qiu et al., 2012).

Different ultrasound exposure parameters for sonoporation have resulted in different cellular effects (Fan et al., 2014). These parameters include the center frequency of the ultrasound transducer, the acoustic pressure, the pulse length, the pulse repetition

20 frequency, etc. As the number of ultrasound pulses increased, cell viability decreased (Park et al., 2011). Severe cell damage was also associated with the acoustic pressure of the applied pulses. As the acoustic pressure increases, damage increases in a generally linear fashion (Brayman et al., 1999; Qiu et al., 2010). Thus, an ultrasound pulse 
sequence must be designed to fulfill the requirement of both minimum cell damage and maximum desired bioeffect.

Previous studies have utilized a diverse range of experimental setups, ultrasound parameters, microbubble formulations, and cell types which resulted in large variations in

5 delivery rates $(\mathrm{Yu}$ and $\mathrm{Xu}, 2014)$. Intracellular drug delivery in human umbilical vein endothelial cells (HUVECs) was shown to depend on the experienced shear conditions, with the observed rates being below $10 \%$ in the physiologically relevant scenario (Park et al., 2011). Previous research which focused on sonoporation applied to modulate gene expression in HUVECs led to a transfection rate of $25 \%$ (Guo et al., 2004). More

10 recently, GFP reporter plasmids were delivered to HEK294 cells with an efficiency of up to $80 \%$ (Tlaxca et al., 2010). A similar delivery rate was achieved at high pressures in the DNA transfection of MCF-7 cells (Qiu et al., 2010). However, high delivery rates were accomplished at the expense of cell viability, with extended cell death being observed at high pressure sonication $\left(\mathrm{P}_{\mathrm{pk}-\mathrm{neg}}>500 \mathrm{kPa}\right)$; in contrast, low acoustic pressures $\left(\mathrm{P}_{\mathrm{pk}-\mathrm{neg}}<\right.$

$15100 \mathrm{kPa}_{\text {pk-neg }}$ ) were proved to be safer but resulted in transfection efficiency below $15 \%$ (Qiu et al., 2012). A common feature of most of the aforementioned studies is that experiments were conducted in the absence of flow.

The presence of flow is associated with various physical effects such as shear stress, since endothelial cells in the human body are exposed to blood flow.

20 Hemodynamic effects largely influence cells exposed to blood flow (Garcia-Cardeña et al., 2001). Furthermore, shear stress increases endothelial cell adhesion to the substratum (Dardik et al., 1999) and alters gene expression and thereby its phenotype (Morita et al., 1993). Endothelial cells are predominantly affected by flow conditions, both in terms of 
function (Balcells et al., 2010; Sato and Ohashi, 2005) and morphology (Rouleau et al., 2010). Cells cultivated under shear stress conditions prior to sonoporation showed lower percentage responses of drug uptake in comparison to static cultured cells after sonication (Park et al., 2011), revealing that physiologically-relevant growth conditions render 5 endothelial cell membrane less prone to ultrasound-mediated trans-membrane delivery. An additional advantage of the presence of flow is that the ultrasound pulse sequence can be modified in order to uniformly distribute the acoustic cavitation activity within the focal volume (Pouliopoulos et al., 2014). Furthermore, the cavitation nuclei kinetics within the focal volume can be adjusted by varying the pulse-off time (Goertz et al., 10 2010). Although it has been previously shown that the employed pulse sequence parameters greatly influence the cell viability and membrane permeability (Karshafian et al., 2009), there has been no feasibility study on a physiologically relevant flow setup.

The aim of our study was (1) to determine whether acoustic cavitation-mediated trans-membrane molecular delivery can be produced at low acoustic pressures during 15 physiologically relevant flow conditions and (2) to rationally modify the ultrasound parameters in order to maximize trans-membrane delivery efficacy while minimizing cellular damage. In this feasibility study, we will demonstrate a high cavitation-mediated trans-membrane delivery rate, coupled with enhanced cell viability, using vascular endothelial cells in vitro under arterial flow conditions. 


\section{METHODS}

In vitro cell culture

Vascular endothelial cells were used due to their importance in cardiovascular

5 pathologies and due to the feasibility of targeting them in vivo for the treatment of atherosclerosis. The human umbilical vein cell line (EA.hy926; CRL-2922; ATCC Manassas, VA, USA) was used in the experiments. The cells were cultured in phenol red free DMEM medium (11880-036; Invitrogen, Life Technologies, Carlsbad, CA, USA) and were maintained in a humidified incubator at standard conditions $\left(37^{\circ} \mathrm{C}, 5 \% \mathrm{CO}_{2}\right)$.

10 The medium was renewed every $2-3$ days. The EA.hy926 cells were sub-cultured in 75 $\mathrm{cm}^{2}$ flasks in the absence of flow until they reached $70-90 \%$ confluence.

\section{Microbubbles manufacturing}

Microbubbles were manufactured in-house following previously described

15 protocols (Banerjee et al., 2012; Pouliopoulos et al., 2014) and were composed of a phospholipid shell and a perfluorobutane gas core. The microbubble shell consisted of three lipids (Avanti Polar Lipids Inc., AL, USA) originally in powder form: DPPC (Dipalmitoylphosphatidylcholine), DPPA (Dipalmitoylphosphatidic acid), and DPPEPEG 5000 (dipalmitolyphosphatidylethanolamine-PEG5000) which were mixed and

20 diluted with glycerol (5\% v/v) and saline $(80 \% \mathrm{v} / \mathrm{v})$. Before each experiment, perfluorobutane gas (FluoroMed L.P., Texas, USA) was introduced into the phospholipid mixture and the solution was mechanically activated using an amalgamator (Synergy Electronics, Scottsdale, AZ, USA). The microbubbles were counted and sized using 
optical microscopy and a previously described image processing technique (Sennoga et al., 2010). The average microbubble concentration was found to be $7.3 \pm 4 \times 10^{9}$ microbubbles/ml with an average size of $1.62 \pm 0.87 \mu \mathrm{m}$, while excluding bubbles with diameters below $0.5 \mu \mathrm{m}$. The clinically recommended dose for Definity contrast agents is

$5 \quad 0.02 \mathrm{ml} / \mathrm{kg}$ (Unger et al., 2004). Our in-house manufactured microbubbles were diluted to match this concentration. Taking into consideration an average body weight of $70 \mathrm{~kg}$ and a blood volume of $5 \mathrm{~L}$, the acceptable equivalent dose would be equal to a concentration of approx. $2 \times 10^{6}$ microbubbles/ml. Microbubble concentrations at $1 \mathrm{x}$ and $50 \mathrm{x}$ the clinically recommended dose were used.

In vitro flow setup

Our in vitro experiments were conducted in physiologically relevant flow conditions. EA.hy926 cells were seeded in sterile collagen treated flow chambers (collagen IV coated $\mu$-Slide $\mathrm{I}^{0.4}$, Ibidi, Martinsried, Germany) at a concentration of $1.5 \times$

$1510^{5}$ cells $/ \mathrm{ml}\left( \pm 4 \times 10^{2}\right.$ cells $\left./ \mathrm{ml}\right)$ and grown as a monolayer for 24 hours. Flow was applied to the adherent cells for this time period in order to restore their physiological phenotype and functionality (Li et al., 2005). The cell chamber outlet was connected to tubing that ran into a flask containing the cell culture medium, propidium iodide (PI), and the microbubbles. The flask outlet was connected to a tube that ran into the inlet of the

20 chamber, which in turn was attached to a peristaltic pump (MPII Mini-Peristaltic Pump; Harvard Apparatus, Holliston, MA, USA) that maintained a fluid velocity of $12.5 \mathrm{~cm} / \mathrm{s}$ and a flow rate of $24.5 \mathrm{ml} / \mathrm{min}$ (figure 1(a)). The velocity was chosen to match the 
measured blood speed in the vicinity of atherosclerotic plaque formed at a carotid artery bifurcation (Zarins et al., 1983).

\section{Cell Staining}

In the present study, calcein-AM and PI were used to track cell viability and drug delivery, respectively. Calcein-AM (C3099; Life Technologies, Carlsbad, CA, USA) is a cell permeable and non-fluorescent compound that is hydrolyzed by intracellular esterase into a green fluorescent anion with excitation and emission wavelengths of 495 and 516 $\mathrm{nm}$, respectively. The presence and activity of these esterases imply cell viability. PI

10 (P4170-100MG; Sigma-Aldrich, St. Louis, MO, USA) is a red fluorescent dye with an excitation wavelength of $536 \mathrm{~nm}$ and an emission wavelength of $617 \mathrm{~nm}$; fluorescent emission is triggered upon its binding with the double stranded nucleic acids. PI is normally impermeable to the cell membrane of viable cells despite its relatively small size (668.4 Da) and has been previously used as sonoporation indicator (Fan et al., 2012;

15 Kooiman et al., 2011; Park et al., 2011; van Wamel et al., 2006). Successful transmembrane delivery would thus facilitate the passage of PI into the nucleus of the cell while maintaining viability and would result in the co-localization of green and red fluorescence. Calcein-AM at a concentration of $1 \mu \mathrm{M}$ per chamber was added $20 \mathrm{~min}$ prior to sonication to track cell viability and the cell monolayer was imaged 15 minutes

20 afterwards. After imaging the monolayer, the chamber was washed with phenol red free DMEM to remove any excess calcein-AM and PI was introduced at a concentration of 25 $\mu \mathrm{g} / \mathrm{ml}$ 
Focused ultrasound system

A single-element spherical-segment FUS transducer with a center frequency of 0.5 MHz (active diameter: 64mm, FWHM: 5.85mm, focal length: 62.6mm, part number: H107; Sonic Concepts Inc., Bothel, WA, USA) was used to drive acoustic cavitation

5 activity while an inserted focused 7.5 MHz single-element focused transducer (diameter: 12.7mm, focal length: 60mm, part number: U8423589; Olympus Industrial, Essex, UK) served as a passive cavitation detector (PCD) and was used to passively detect microbubble acoustic emissions (figure 1(a)). The two transducers were co-axially aligned so that their focal volumes overlapped. A $0.2 \mathrm{~mm}$ polyvinylidene fluoride (PVDF)

10 hydrophone (Precision Acoustics Ltd., Dorchester, UK) was used to determine the focal regions and the emitted acoustic pressures. The attenuation of the Ibidi chamber inclusive of both the front and back wall was found to be $19.3 \%$. This attenuation value is likely even less within the chamber.. The cell flow chamber was positioned within the focal volume of the $0.5 \mathrm{MHz}$ transducer so that its focal point was placed over the target layer

15 of cells (figure 1(a)). The ultrasound transducers and flow chamber were placed in a large tank containing deionized and degassed water maintained at $37^{\circ} \mathrm{C}$ to imitate in vivo conditions.

A waveform generator (Agilent Technologies 33500B Series, Santa Clara, CA, USA) connected to a $50 \mathrm{~dB}$ power amplifier (Precision Acoustics Ltd, Dorchester, UK) 20 and a matching network (SonicConcepts Inc., Bothel, WA, USA) was used to drive the 0.5 MHz FUS transducer. Ultrasound parameters, such as peak negative acoustic pressure $\left(\mathrm{P}_{\mathrm{pk}-\mathrm{neg}}\right)$, pulse length $(\mathrm{PL})$, pulse repetition frequency $(\mathrm{PRF})$, and number of pulses $\left(\mathrm{N}_{\mathrm{p}}\right)$ were set for every experiment using Matlab (The Mathworks, Natick, MA, USA), which 
controlled the emission and acquisition processes. The $7.5 \mathrm{MHz}$ PCD was connected to a 1.25 MHz high-pass filter (part number: ZFHP-1R2-S+; Mini-Circuits, Surrey, UK) or a 5MHz high-pass filter (part number: MH-500P-C-P; Allen Avionics, Mineola, NY, USA) and a $28 \mathrm{~dB}$ pre-amplifier (Stanford Research Systems, Sunnyvale, CA, USA), to cut off

5 the fundamental frequency signal and amplify low amplitude acoustic emissions specific to microbubble cavitation respectively. An 8-bit oscilloscope (DPO3014, Tektronix, Inc. OR, USA) was used to capture the acoustic signal at a sampling rate of $50 \mathrm{MSa} / \mathrm{s}$.

\section{Experimental protocol}

10 Confluency, viability, and drug delivery into EA.hy926 cells were measured before and after sonication using a Zeiss Palm MicroBeam microscope (Zeiss Palm MicroBeam, Carl Zeiss AG, Oberkochen, Germany). Fifteen minutes prior to sonication, calcein-AM was added to the flow chamber to evaluate cell confluency. Images were captured at five positions throughout the cell chamber: near the inlet, midpoint between the inlet and the

15 sonicated region, the sonicated region, midpoint between the sonicated region and the outlet, and near the outlet (figure 1(b)). At each zone, two images were captured at exposure times of 15 and $30 \mathrm{~ms}$ to acquire images with acceptable intensity. Immediately after sonication, the cells were imaged serially until trans-membrane drug delivery was observed or until 2 hours after sonication. Between each image acquisition, the treated

20 cells were moved to a humidified incubator and kept in standard conditions $\left(37^{\circ} \mathrm{C}, 5 \%\right.$ $\mathrm{CO}_{2}$ ) without being exposed to flow.

A total of 18 different sets of ultrasound parameters ( $\mathrm{P}_{\mathrm{pk}-\mathrm{neg}}$ : 40-160kPa, PL: 10-500 cycles, $\mathrm{N}_{\mathrm{p}}$ : 10-600) were evaluated in our experiments in two different phases: a rough 
exploration of ultrasound parameters that would produce distinct bioeffects, and a quantitative study on the effects of the best parameter set discovered that could enhance trans-membrane drug delivery without increasing damage. In the first phase, the systematic exploration of parameters - by modification of one parameter at a time - was

5 considered, because it could provide insight into the influence of each parameter. However, modifying a single ultrasound parameter at a time does not imply modifying a single characteristic of microbubble dynamics (e.g., a type, a magnitude, a spatial distribution, and a temporal duration of cavitation; and microbubble velocities and aggregation due to radiation forces). Thus, we sought modification of several parameters

10 simultaneously based on evidence provided from unsuccessful parameters to produce the desired bioeffect. We have included in this paper only 3 sets that described the wide range of bioeffects that were observed. In the final phase, we quantitatively characterised the effects of the best parameter set that achieved the objective. The trans-membrane delivery with the optimal parameter set was compared to three controls. The first control

15 experiment tested the effect of flow only on cells, without FUS or microbubbles. The second control tested the effect of flow and FUS, while the third control tested the effect of the presence of flow and microbubbles only.

\section{Analysis of cell viability}

The fluorescent cell images of calcein-AM and PI were processed and analyzed using ImageJ (1.47v, National Institute of Health, USA). The image was converted into a binary format and any merged cells were manually separated. The total number of cells in the image and the position of each single-cell area were determined using built-in ImageJ 
functions. The aforementioned process was performed for both green and red fluorescent images, corresponding to the calcein-AM and PI fluorescent emissions respectively. The overlap of the two colors was counted using ImageJ and the final outcome represented the number of successful trans-membrane delivery without cell death.

\section{Post-sonoporation image analysis}

Three distinct types of cells within the focal area were identified in the postsonoporation image. Type A cells were defined as viable non-sonoporated cells, type B as non-viable sonoporated cells, and type $\mathrm{C}$ as viable and sonoporated cells. Non-sonicated

10 cells served as a control. For the successful sonoporation experiments, calcein-AM and PI channels were segmented into two different intensity classes in Matlab, namely upper and lower, using arbitrary thresholds determined by qualitative interpretation of the intensity histograms. The upper class contained pixels with intensity above the defined threshold (characterized as calcein-AM or PI positive), while the lower class contained pixels with

15 intensity values below the threshold (characterized as calcein-AM or PI negative, i.e. noise). Type A cells had intensity values in the upper green and lower red class, type B cells had values in the upper red and lower green class, and type C cells' fluorescence lied within the upper class both in the calcein-AM and the PI channel. Thirty randomly selected cells of each type were segmented, labeled and independently analyzed in terms

20 of their fluorescence intensity. Finally, lateral intensity profiles across a cell of each type were extracted to compare the spatial evolution of calcein-AM and PI fluorescence.

Analysis of Cavitation Emissions 
Acoustic emissions captured using the $7.5 \mathrm{MHz}$ PCD were analyzed in order to monitor the activity of the flowing cavitation nuclei. Analysis was conducted in terms of energy and spectral content of the microbubble emissions. Energy values were determined by integrating the time-domain PCD signal over time and were corrected for

5 the electronic noise contribution (Pouliopoulos et al., 2014). Acoustic emissions were also spectrally analyzed in order to elucidate the temporally resolved microbubble activity during the entire sonication duration.

\section{Statistical Analysis}

10 Statistical tests were performed to ensure statistical significance of our results. The data sets for the different cell types were compared using one-way analysis of variance (ANOVA) tests followed by post-hoc Bonferroni analysis, both for the calcein-AM and the PI fluorescence. The significance level adopted was $p<0.001$. All measurements are expressed as mean \pm standard deviation. 


\section{RESULTS}

\section{Evaluation of ultrasound parameters for sonoporation during flow}

In order to identify ultrasound parameters suitable for trans-membrane delivery

5 under high flow rates, we first performed a rough parametric search and evaluated performance using qualitative observations. A series of pilot experiments were performed during the evaluation procedure of our flow system, using typical sonoporation ultrasound parameters $\left(\mathrm{P}_{\mathrm{pk}-\mathrm{neg}}\right.$ : 40-160kPa, PL: 10-500 cycles, $\mathrm{N}_{\mathrm{p}}$ : 10-600). Our initial selection of ultrasound parameters was based on previous studies without flow, which

10 have shown that higher acoustic pressures increase not only the desired trans-membrane delivery efficiency, but also the unwanted cellular damage (Brayman et al., 1999; Qiu et al., 2012). Studies have also shown that inertial cavitation is responsible for the decreased cell viability (Newman and Bettinger, 2007). Yet there have been reports of successful trans-membrane delivery while maintaining cell viability, but this has been limited to a

15 very small percentage of the total sonicated cell population (Lammertink et al., 2014; Qiu et al., 2012, 2010). Our rationale in the design of ultrasound pulse sequences was that these particular cells were sonoporated due to a specific range of the locally experienced cavitation dynamics, which can be controlled by the ultrasound pulse shape. If this type of cavitation was identified, then it could be replicated over a larger number of cells by

20 exploiting the presence of flow and adjusting the pulse repetition frequency accordingly. In this paper we will present three sets of parameters that summarize the distinct observed bioeffects and our pulse shape and sequence design rationale. 
The first pulse shape evaluated was characterized by a center frequency $\left(f_{c}\right)$ of 0.5 $\mathrm{MHz}$, a $\mathrm{P}_{\mathrm{pk}-\mathrm{neg}}$ of $110 \mathrm{kPa}$, and a PL of 100 cycles. This pulse was emitted 50 times at a PRF of $0.2 \mathrm{~Hz}$. Taking into account the high flow velocity of the circulating solution $(12.5 \mathrm{~cm} / \mathrm{s})$ and the lateral FWHM of our therapeutic transducer $(5.85 \mathrm{~mm})$, complete 5 reperfusion of the focal volume was expected to occur in approximately $50 \mathrm{~ms}$. Thus, a PRF of $20 \mathrm{~Hz}$ or lower would suffice for cavitation nuclei replenishment. The PRF of $0.2 \mathrm{~Hz}$ was selected to allow enough time $(\approx 5 \mathrm{sec})$ for the recording and transfer of the acoustic emissions data to the PC. Before sonication, it was confirmed that cells had covered the entire focal area and that calcein-AM had been adequately uptaken (figure

10 2(a)). During sonication with the initial parameters and circulation of microbubbles at $1 \mathrm{X}$ the clinical dose, cells were dislodged from the flow chamber, while causing an increase in PI without co-localisation of calcein-AM, thus indicating cell death (figure 2(b)).

In order to avoid cell death and dislodgement we reduced the pressure and pulse length ( $f_{\mathrm{c}}: 0.5 \mathrm{MHz}, \mathrm{P}_{\mathrm{pk} \text {-neg: }} 75 \mathrm{kPa}$, PL: 50 cycles, PRF: $0.2 \mathrm{~Hz}$, and $\mathrm{N}_{\mathrm{p}}: 50$ ), while

15 maintaining the microbubble concentration at $1 \mathrm{x}$ the clinical dose. The most striking observations were that no or few cells were dislodged and that the number of dead type B cells was significantly reduced. Interestingly, there was a total of 4 type $\mathrm{C}$ cells with colocalisation of green and red fluorescence within the focal area (figure 2(c)), thus indicating a limited trans-membrane delivery of PI $(<1 \%)$ while maintaining cell viability 20 (>99\%). This result indicated that safe sonoporation was feasible if the cell experiences a mild cavitation effect.

Analysis of safe trans-membrane delivery 
We assumed that higher pressures were responsible for the dead cells whilst the type of cavitation which locally resulted in safe trans-membrane delivery was being produced by a very small subset of microbubbles. Therefore, the pressure was further lowered, the number of pulses was increased, and the microbubble concentration was 5 increased to $50 \mathrm{x}$ the clinical dose. Sonication with the adjusted parameters $\left(\mathrm{f}_{\mathrm{c}}: 0.5 \mathrm{MHz}\right.$, $\mathrm{P}_{\text {pk-neg: }} 40 \mathrm{kPa}, \mathrm{PL}: 50$ cycles, PRF: $0.2 \mathrm{~Hz}$, and $\mathrm{N}_{\mathrm{p}}$ : 500) was shown to produce the desired uniform bioeffect, resulting in successful trans-membrane PI delivery with limited cell death. This effect was constrained within the sonicated focal area and was not present in the control areas near the inlet and outlet of the seeded chamber (data not

10 shown). Control experiments with the same sonication parameters but without microbubbles and/or ultrasound (figures 3(a)-3(c) and table 1) confirmed that sonoporation occurred only due to the combined use of microbubbles and focused ultrasound. Following ultrasound exposure, red and green fluorescence co-localization was observed within the focal region (figure 3(e)), surrounded by a sharp boundary of

15 viable cells that did not experience ultrasound exposure (figures 3(d) and 3(f)). Using the pre- and post-sonication images, we measured a drug delivery rate of $62.6 \pm 4.3 \%(n=2)$ and a cell viability of $95 \pm 4 \%$ (table 1$)$.

All three cell types were observed within the sonicated region (figure 3(e)), indicating that despite the presence of flow cavitation activity was not uniformly 20 distributed throughout the focal area. The stochastic nature of the cavitation events led to instances of either unsuccessful (type A) or excess (type B) membrane disruption (figure 4(a)). However the majority of the cells were viable and successfully sonoporated (type C). Intensity profiles revealed that type A and B cells presented PI and calcein-AM 
fluorescence below the noise level respectively, as expected. In contrast, type C cells fluorescence was above the noise level for both channels (figure 4(b)). Type B cell type had a significantly different calcein-AM and PI fluorescence intensity when compared with the Type A control $(p<0.001$, figure $4(\mathrm{c}))$. Similarly, both channels presented

5 statistically significant difference between the type B and type C cells. In contrast, type C cells showed significantly higher intensity levels than type A cells only in the PI channel, with the change in calcein-AM mean intensity being non-significant $(p>0.05$, figure 4(c)). Interestingly, the mean green fluorescence of type A and C cells was approximately $70 \%$ lower than the green fluorescence outside the insonation region which acted as a

10 control (figures 3(d) and 3(f)) but still above the defined threshold. The average intensities of each cell type were insensitive to changes of either the green fluorescence threshold or the total number of the randomly selected cells.

\section{Acoustic emissions from cavitation activity}

15 Analysis of the acoustic emissions from the cavitation activity showed that microbubbles persisted throughout the entire pulse sequence. Following correction for the electronic noise contribution, the energy of the microbubble emissions for the first set of the tested parameters ( $\mathrm{f}_{\mathrm{c}}$ : $0.5 \mathrm{MHz}, \mathrm{P}_{\mathrm{pk}-\mathrm{neg}}$ : $110 \mathrm{kPa}, \mathrm{PL}: 100$ cycles, PRF: $0.2 \mathrm{~Hz}$, and $\mathrm{N}_{\mathrm{p}}$ : 50) showed large inter-pulse variations following a decreasing trend (figure 5(a)), while the

20 energy of the final set of parameters ( $f_{c}: 0.5 \mathrm{MHz}, \mathrm{P}_{\mathrm{pk} \text {-neg: }}: 40 \mathrm{kPa}$, PL: 50 cycles, PRF: 0.2 $\mathrm{Hz}$, and $\mathrm{N}_{\mathrm{p}}$ : 500) was lower and decayed with time (figure 5(c)). This could be due to either the gradual decrease in the circulating microbubble concentration, or the gradual change of the microbubble sizes. In contrast, no detectable emissions were recorded in 
the control experiment without circulating microbubbles. The low peak-rarefactional pressures $\left(\mathrm{P}_{\mathrm{pk}-\mathrm{neg}}\right.$ : 40-110 $\left.\mathrm{kPa}\right)$ were not expected to trigger transient inertial cavitation events. This was confirmed by the spectral analysis for the final parametric set $\left(\mathrm{P}_{\mathrm{pk}-\mathrm{neg}}: 40\right.$ $\mathrm{kPa}$ ), which revealed no broadband emissions in the frequency spectrum. Instead, the 5 persistent presence of low order harmonics indicated the dominance of non-inertial cavitation throughout the entire sonication duration (figure 5(d)). On the contrary, the spectrogram of the first sonication parameter set $\left(\mathrm{P}_{\mathrm{pk}-\mathrm{neg}}\right.$ : $\left.110 \mathrm{kPa}\right)$ produced broadband signatures together with higher order harmonics, thus indicating the presence of inertial cavitation (figure 5(b)). 


\section{DISCUSSION}

\section{Enhancement of safe and efficient intra-cellular drug delivery}

The results presented in this paper demonstrate how the approach of incorporating

5 flow in the system is desirable for successful sonoporation. The final parameters that were used ( $\mathrm{f}_{\mathrm{c}}$ : $0.5 \mathrm{MHz}, \mathrm{P}_{\mathrm{pk}-\mathrm{neg}}$ : $40 \mathrm{kPa}$, PL: 50 cycles, PRF: $0.2 \mathrm{~Hz}$, and $\mathrm{N}_{\mathrm{p}}: 500$ ) with a concentration 50X the clinical dose showed a drug delivery rate of $62.6 \pm 4.3 \%$ and a cell viability of $95 \pm 4 \%$ (table 1). These parameters were deemed to produce the highest trans-membrane molecular delivery efficiency while avoiding cell death from within the

10 limited set of parameters evaluated in our exploration protocol. Cells dislodgement from the targeted area was observed with the initial sonication parameters $\left(\mathrm{f}_{\mathrm{c}}: 0.5 \mathrm{MHz}, \mathrm{P}_{\mathrm{pk} \text {-neg: }}\right.$ : $110 \mathrm{kPa}$, PL: 100 cycles, PRF: $0.2 \mathrm{~Hz}$, and $\mathrm{N}_{\mathrm{p}}$ : 50), thus it was inferred that limited low magnitude inertial cavitation has occurred (figure 5(b)), as shown in previous studies (Brayman et al., 1999). Dislodgement is not likely to occur in vivo and is an artefact of

15 our in vitro system. Our results are physiologically relevant at lower pressures where cell detachment did not occur and this constitutes the majority of the parameters we explored. Consequently, the $\mathrm{P}_{\mathrm{pk} \text {-neg }}$ was decreased to $40 \mathrm{kPa}$, the number of cycles was decreased from 100 to 50, the number of pulses was increased from 50 to 500 , and the cavitation nuclei number was increased 50 times. The primary reason for the reduction of the

20 acoustic pressure and the number of cycles was to achieve stable cavitation that is moderate and safe to the seeded cells. The rationale behind using 500 pulses was to increase the total number of interactions between the microbubbles undergoing the ideal cavitation dynamics and the cell monolayer. Finally, the increase in the microbubble 
concentration ensured an adequate microbubble number, uniformly distributed over the cell monolayer.

The selected set of parameters led to a high delivery rate combined with high cell viability (figure 3(e) and table 1). Co-localization of green and red fluorescence was 5 proved to be an effect of the synergistic action of the FUS and the flowing microbubbles, as indicated by the control experiments (figures 3(a)-3(c)) and the sharp boundary observed between the focal region and its surroundings (figures 3(d)-3(f)). By assessing figure 3(e), we identified three distinct types of cells, namely A, B, and C, according to their uptake of calcein-AM and PI. We hypothesize that PI is entering through pores

10 created during or after the interaction between the microbubbles undergoing cavitation and the neighboring cell membranes. It is likely that calcein-AM has completely leaked out of the severely disrupted cell membrane in type B cells, thus reducing the total green fluorescent emission within the cytoplasm to levels below the arbitrary threshold. In type C cells, the limited breach of the cell membrane integrity resulted in a significant increase

15 in the PI fluorescence levels when compared to type A, while a percentage of the cleaved calcein-AM molecules leaked out, yet the average green fluorescence was maintained at the same levels with type A ( $p>0.05)$. Moderate cavitation activity (figure $5(\mathrm{~d}))$ has thus been shown sufficient for successful PI delivery. However, a $70 \%$ reduction was observed in the mean green fluorescence intensity within the sonicated region when 20 compared to the non-sonicated region even in type A cells (figures 3(d), 3(f), 4(c)). A possible mechanism is that the physiology of viable cells is altered due to the external mechanical excitation, leading to a reduction in the emitted green fluorescence. Examining the origin of this reduction is part of our future work. We hypothesize that the 
local inhomogeneity of the microbubble population characteristics, i.e. the variable number and size distribution, is the dominant factor contributing towards the production of the three distinct cell types. Other factors include the variability of the locally experienced ultrasound pressure, and the cycle stage of each cell which may have an 5 effect on the robustness and mechanical properties of each membrane.

It was also noted that time plays an important role for the PI uptake efficiency. Colocalization of the red and green fluorescence was observed approximately 2 hours after sonication, indicating a time interval between mechanical agitation of the cell membrane and successful delivery through the membrane. The time delay is correlated with the

10 relatively slow PI uptake from viable cells with a transiently permeable membrane and can be due to triggered endocytosis (Geers et al., 2011; Meijering et al., 2009), in contrast with dead cells with severely breached membrane that rapidly equilibrate with the surrounding medium's PI concentration to give bright red fluorescence (Djuzenova et al., 1996).

15 The biological mechanism responsible for our findings is related to the cellular intake mechanism. It has been shown that membrane pore formation not only allows direct molecule permeation into the cytoplasm, but can also trigger transient intracellular defense mechanisms that can be responsible for the uptake of macromolecules (Fan et al., 2010). Enhanced drug delivery of large molecules (>150 kDa) was previously shown to

20 be a result of ultrasound-induced endocytosis (Meijering et al., 2009), which may be the cause of the delayed detection of PI fluorescence (2 hours post-sonication). PI could be delivered through endocytosis, but its small molecular weight (668.4 Da) may allow it to be transported through other routes which larger compounds cannot utilize (e.g., through 
mechanically-induced pores)(Zhao et al. 2008). Our results were consistent with previous studies that verified enhanced cell membrane permeability of endothelial cells due to ultrasound exposure and microbubbles (van Wamel et al., 2006). Thus, we hypothesize that the observed trans-membrane delivery is a result of a passive transport mechanism

5 coupled with non-specific membrane poration or limited physical disruption of the cell membrane due to the mechanical interaction with the oscillating microbubbles (Fan et al., 2010; Prentice et al., 2005) which may trigger a cascade of events eventually leading to apoptosis (Zhong et al., 2013).

\section{Acoustic Cavitation characterization}

One of the goals of this study was to understand the cavitation mechanism that is responsible for optimum bioeffects. Inertial cavitation occurs when microbubbles respond to the acoustic field in a highly nonlinear manner that can be yielded with high temperatures and pressures (Apfel, 1997; Dalecki, 2004). This type of cavitation has been

15 related to thermal mechanisms that can also result with chemical processes. Most importantly, it has been shown that cell lysis can occur from high magnitude inertial cavitation (Miller and Thomas 1996). Previous findings have shown how inertial cavitation produces violent shock waves that result in erosion of cell monolayers (Apfel 1997; Brayman et al. 1999; Newman and Bettinger 2007). A similar effect is shown in

20 the present work (figures 2(b) and 5(a)-(b)), where low magnitude inertial cavitation activity led to extensive cell detachment and cell death at the initial parametric set $\left(f_{c}: 0.5\right.$ MHz, $\mathrm{P}_{\text {pk-neg: }} 110 \mathrm{kPa}, \mathrm{PL}: 100$ cycles, PRF: $0.2 \mathrm{~Hz}$, and $\mathrm{N}_{\mathrm{p}}$ : 50). The presence of not only higher order harmonics, but also broadband signal suggested that there is a mixed 
distribution of cavitation modes (figure 5(b)). Based on this data, it is likely that cavitation activity spans from high magnitude non-inertial cavitation up to low magnitude inertial cavitation activity. Considering that the microbubble size distribution is polydisperse, that we are using a low center frequency of $0.5 \mathrm{MHz}$, and that our pulse

5 lengths are long (greater than or equal to 50 cycles), this finding is within a reasonable range as theoretical cavitation thresholds shown previously (Apfel and Holland 1991). In addition, previous work with this microbubble formulation has shown that pulses emitted at a $0.5 \mathrm{MHz}$ center frequency and in this pressure regime $\left(\mathrm{P}_{\mathrm{pk}-\mathrm{neg}}: 147 \mathrm{kPa}\right)$ can produce a mixture of high magnitude non-inertial and low magnitude inertial cavitation

10 (Pouliopoulos et al. 2014), which is in agreement with the current study.

In contrast, the microbubble acoustic signal analysis of the final parametric set $\left(f_{c}\right.$ : $0.5 \mathrm{MHz}, \mathrm{P}_{\text {pk-neg: }} 40 \mathrm{kPa}$, PL: 50 cycles, PRF: $0.2 \mathrm{~Hz}$, and $\mathrm{N}_{\mathrm{p}}$ : 500) showed that stable cavitation was the dominant cavitation mode during the ultrasound exposure (figure 5(d)). The low pressure and pulse length ensured safe and efficient drug delivery (figure

$153(\mathrm{e})$ and table 1), confirming previous results indicating that intact and stably oscillating microbubbles are beneficial for the sonoporation efficiency (Fan et al., 2012; van Wamel et al., 2006).

The decay in the emitted energy (figures 5(a) and 5(c)) may be due to either the decrease of the circulating microbubble concentration or the gradual reduction of the 20 microbubble sizes, originating from the natural or acoustically driven diffusion of the gas core through the lipid shell (Borden and Longo, 2002). The microbubble size distribution could shift towards smaller sizes, leading to a decrease of the magnitude of the radiated microbubble acoustic emissions (Radhakrishnan et al., 2013). Stable cavitation, i.e. 
moderate microbubble oscillations, is crucial for the safety and efficacy of drug delivery via sonoporation, since the violent effects occurring within the transient cavitation regime such as microbubble fragmentation and jet stream formation would cause irreversible damage to the cell membrane and increase the cell death (figures 2(b) and 5(b)).

\section{Limitations of the study}

Despite the successful sonoporation effects, the major limitation of the present study is the variation of the mechanical effect on each individual cell, yielding three distinct types of cells within the focal volume (figure 3(e)). In an ideal sonoporation

10 experiment, all cells would experience the same amount of mechanical agitation by the nearby oscillating microbubbles, producing only viable and sonoporated cells, i.e. type C cells. The effect of heterogeneous molecular uptake by sonicated cells has been described before (Guzmán et al., 2001a, 2001b) and constitutes a common limitation in sonoporation experiments. In our current studies, every effort is being made to reduce the

15 variability and yield uniform sonoporation effects across the focal volume, by modifying the characteristics of the ultrasound pulse shape and sequence and standardizing the experimental procedure.

In order to achieve higher trans-membrane delivery efficiency, we decided to increase the number of cavitation nuclei, since the previously tested concentration yielded

20 a lower number of cells with evidence of trans-membrane delivery with similar sonication parameters. However the final concentration is above the recommended dose for imaging applications (Unger et al. 2004), and may also cause shadowing effects which could lead to variations of the in situ pressure experienced by the cell monolayer. 
Therefore in our future studies the cavitation nuclei concentration will be gradually reduced and new pulse shapes and sequences will be designed in order to achieve the same bioeffect with the clinically approved concentration. One possible solution is to significantly increase the number of pulses emitted per unit time (i.e., increase the pulse

5 repetition frequency), which would be effective due to the high microbubble replenishment rate of arterial flow. Finally, despite the improvements in the physiological relevance of our in vitro model, there remain several key differences with the in vivo conditions. Unlike the uninterrupted cell lining present in a vessel wall, here the cell monolayer was incomplete, subjected to flow only for 24 hours before sonication and

10 kept in static conditions post-sonication. It is thus likely that the in vitro functionality and morphology of endothelial cells may not be identical to the in vivo scenario (Balcells et al., 2010; Rouleau et al., 2010; Sato and Ohashi, 2005). Although our flow system sustained a physiologically relevant flow rate, the hydrodynamic pressure was not controlled and this could affect the cavitation dynamics and the cell responses to

15 mechanical stimuli. In terms of mechanical and acoustic properties, our flow chambers did not match the stiffness or the acoustic impedance of the vessel wall respectively. Moreover, the properties of blood and pure saline differ (e.g., viscosity), thus a different magnitude of shear stress is expected in vivo. Also, microbubble dynamics are likely to be affected due to the presence of other molecules (e.g., proteins) and cells (e.g.,

20 erythrocytes). For example, the pressure threshold for inertial cavitation is higher in blood than in water (Apfel and Holland 1991). A different acoustic response and reflection pattern are expected within the real vasculature. Thus, our future work involves 
in vivo implementation of the reported pulse sequences, in order to test the safety and efficacy of the ultrasound-enhanced intracellular drug delivery in atherosclerotic vessels.

Our approach to exploring the multi-dimensional ultrasonic and environmental parametric space was based on the accumulation of empirical evidence and adjusting the

5 next series of evaluation parameters in response to this. Simultaneous modification of several parameters allowed the ability to rapidly traverse the complex and broad landscape of achievable microbubble dynamics. However, the limitation with this approach is that it does not allow us to ascertain the influence of each of these parameters. Modification of a single parameter at a time could help provide better insight

10 into these dependencies. Regardless, we were able to achieve a bioeffect of an enhanced percentage of cells with trans-membrane delivery without exacerbating damage using our approach. A more comprehensive evaluation of parametric dependencies will be conducted in our future work. 


\section{CONCLUSION}

Our ultrasound pulse shape and sequence in the presence of specific microbubble and physiological parameters resulted in a intracellular drug delivery rate of $62.6 \pm 4.3 \%$

5 combined with cell viability of $95 \% \pm 4.2 \%$, thus demonstrating the highest reported efficacy and safety during flow conditions and for endothelial cells. Cavitation analysis and the use of low pressures suggested that inertial cavitation was absent throughout the entire sonication with the selected ultrasound parameters and that stable cavitation was primarily responsible for the delivery. Flow and designing ultrasound parameters to

10 exploit its presence was essential for producing high sonoporation efficiency without extensive cellular damage. This work lays the foundation for future work on the design and optimization of ultrasonic pulse shapes and sequences during flow conditions.

Acknowledgements - This project was supported by the Royal Society Research Grant 2014

15 (RG130795). The authors of this study would like to especially thank Mrs. Shanas Choudhury and Mr. Gary Jones for their technical assistance. 


\section{REFERENCES}

Apfel, R., 1997. Sonic effervescence: A tutorial on acoustic cavitation. J. Acoust. Soc. Am. 101, 1227-1237.

5 Apfel, R.E., Holland, C.K., 1991. Gauging the likelihood of cavitation from short-pulse, low-duty cycle diagnostic ultrasound. Ultrasound Med. Biol. 17, 179-85.

Balcells, M., Martorell, J., Olivé, C., Santacana, M., Chitalia, V., Cardoso, A.A., Edelman, E.R., 2010. Smooth muscle cells orchestrate the endothelial cell response to flow and injury. Circulation 121, 2192-9.

10 Banerjee, B., McKeown, K.R., Skovan, B., Ogram, E., Ingram, P., Ignatenko, N., PaineMurrieta, G., Witte, R., Matsunaga, T.O., 2012. Ultrasound imaging of the mouse pancreatic duct using lipid microbubbles. Proc. SPIE.

Borden, M.A., Longo, M.L., 2002. Dissolution Behavior of Lipid Monolayer-Coated, Air-Filled Microbubbles: Effect of Lipid Hydrophobic Chain Length. Langmuir 18, 9225-9233.

Brayman, A.A., Lizotte, L.M., Miller, M.W., 1999. Erosion of artificial endothelia in vitro by pulsed ultrasound: acoustic pressure, frequency, membrane orientation and microbubble contrast agent dependence. Ultrasound Med. Biol. 25, 1305-20.

Choi, J., Coussios, C., 2012. Spatiotemporal evolution of cavitation dynamics exhibited by flowing microbubbles during ultrasound exposure. J. Acoust. Soc. Am. 132. 
Chomas, J.E., Dayton, P., May, D., Ferrara, K., 2001. Threshold of fragmentation for ultrasonic contrast agents. J. Biomed. Opt. 6, 141-50.

Church, C., Carstensen, E., 2001. "Stable" inertial cavitation. Ultrasound Med. Biol. 27, $1435-1437$.

5 Cosgrove, D., 2006. Ultrasound contrast agents: an overview. Eur. J. Radiol. 60, 324-30.

Dalecki, D., 2004. Mechanical bioeffects of ultrasound. Annu. Rev. Biomed. Eng. 6, $229-48$.

Dardik, A., Liu, A., Ballermann, B.J., 1999. Chronic in vitro shear stress stimulates endothelial cell retention on prosthetic vascular grafts and reduces subsequent in 10 vivo neointimal thickness. J. Vasc. Surg. 29, 157-67.

Djuzenova, C.S., Zimmermann, U., Frank, H., Sukhorukov, V.L., Richter, E., Fuhr, G., 1996. Effect of medium conductivity and composition on the uptake of propidium iodide into electropermeabilized myeloma cells. Biochim. Biophys. Acta 1284, 14352.

15 Fan, Z., Chen, D., Deng, C.X., 2013. Improving ultrasound gene transfection efficiency by controlling ultrasound excitation of microbubbles. J. Control. Release 170, 40113. 
Fan, Z., Chen, D., Deng, C.X., 2014. Characterization of the dynamic activities of a population of microbubbles driven by pulsed ultrasound exposure in sonoporation. Ultrasound Med. Biol. 40, 1260-72.

Fan, Z., Kumon, R.E., Park, J., Deng, C.X., 2010. Intracellular delivery and calcium transients generated in sonoporation facilitated by microbubbles. J. Control. Release $142,31-9$.

Fan, Z., Liu, H., Mayer, M., Deng, C.X., 2012. Spatiotemporally controlled single cell sonoporation. Proc. Natl. Acad. Sci. 109 , 16486-16491.

Ferrara, K., Pollard, R., Borden, M., 2007. Ultrasound microbubble contrast agents: fundamentals and application to gene and drug delivery. Annu. Rev. Biomed. Eng. $9,415-47$.

Flynn, H.G., 1982. Generation of transient cavities in liquids by microsecond pulses of ultrasound. J. Acoust. Soc. Am. 72, 1926.

Garcia-Cardeña, G., Comander, J., Anderson, K.R., Blackman, B.R., Gimbrone, M.A., 2001. Biomechanical activation of vascular endothelium as a determinant of its functional phenotype. Proc. Natl. Acad. Sci. U. S. A. 98, 4478-85.

Geers, B., Lentacker, I., Alonso, A., Sanders, N.N., Demeester, J., Meairs, S., De Smedt, S.C., 2011. Elucidating the mechanisms behind sonoporation with adeno-associated virus-loaded microbubbles. Mol. Pharm. 8, 2244-51. 
Goertz, D.E., Wright, C., Hynynen, K., 2010. Contrast agent kinetics in the rabbit brain during exposure to therapeutic ultrasound. Ultrasound Med. Biol. 36, 916-24.

Guo, D.-P., Li, X.-Y., Sun, P., Wang, Z.-G., Chen, X.-Y., Chen, Q., Fan, L.-M., Zhang, B., Shao, L.-Z., Li, X.-R., 2004. Ultrasound/microbubble enhances foreign gene expression in ECV304 cells and murine myocardium. Acta Biochim. Biophys. Sin. (Shanghai). 36, 824-31.

Guzmán, H.R., Nguyen, D.X., Khan, S., Prausnitz, M.R., 2001a. Ultrasound-mediated disruption of cell membranes. II. Heterogeneous effects on cells. J. Acoust. Soc. Am. 110, 597-606.

10 Guzmán, H.R., Nguyen, D.X., Khan, S., Prausnitz, M.R., 2001b. Ultrasound-mediated disruption of cell membranes. I. Quantification of molecular uptake and cell viability. J. Acoust. Soc. Am. 110, 588-96.

Helfield, B.L., Goertz, D.E., 2013. Nonlinear resonance behavior and linear shell estimates for Definity ${ }^{\mathrm{TM}}$ and MicroMarker ${ }^{\mathrm{TM}}$ assessed with acoustic microbubble spectroscopy. J. Acoust. Soc. Am. 133, 1158-68.

Hu, Y., Wan, J.M.F., Yu, A.C.H., 2013. Membrane perforation and recovery dynamics in microbubble-mediated sonoporation. Ultrasound Med. Biol. 39, 2393-405.

Karshafian, R., Bevan, P.D., Williams, R., Samac, S., Burns, P.N., 2009. Sonoporation by Ultrasound-Activated Microbubble Contrast Agents: Effect of Acoustic Exposure 
Parameters on Cell Membrane Permeability and Cell Viability. Ultrasound Med. Biol. 35, 847-860.

Kooiman, K., Foppen-Harteveld, M., van der Steen, A.F.W., de Jong, N., 2011. Sonoporation of endothelial cells by vibrating targeted microbubbles. J. Control. Release 154, 35-41.

Lammertink, B., Deckers, R., Storm, G., Moonen, C., Bos, C., 2014. Duration of ultrasound-mediated enhanced plasma membrane permeability. Int. J. Pharm.

Levine, R.A., Teichholz, L.E., Goldman, M.E., Steinmetz, M.Y., Baker, M., Meltzer, R.S., 1984. Microbubbles have intracardiac velocities similar to those of red blood cells. J. Am. Coll. Cardiol. 3, 28-33.

Li, Y.-S.J., Haga, J.H., Chien, S., 2005. Molecular basis of the effects of shear stress on vascular endothelial cells. J. Biomech. 38, 1949-71.

Mehier-Humbert, S., Bettinger, T., Yan, F., Guy, R.H., 2005. Plasma membrane poration induced by ultrasound exposure: implication for drug delivery. J. Control. Release $104,213-22$.

Meijering, B.D.M., Juffermans, L.J.M., van Wamel, A., Henning, R.H., Zuhorn, I.S., Emmer, M., Versteilen, A.M.G., Paulus, W.J., van Gilst, W.H., Kooiman, K., de Jong, N., Musters, R.J.P., Deelman, L.E., Kamp, O., 2009. Ultrasound and microbubble-targeted delivery of macromolecules is regulated by induction of 20 endocytosis and pore formation. Circ. Res. 104, 679-87. 
Miller, D.L., Thomas, R.M., 1996. Contrast-agent gas bodies enhance hemolysis induced by lithotripter shock waves and high-intensity focused ultrasound in whole blood. Ultrasound Med. Biol. 22, 1089-95.

Morgan, K.E., Allen, J.S., Dayton, P.A., Chomas, J.E., Klibaov, A.L., Ferrara, K.W., 2000. Experimental and theoretical evaluation of microbubble behavior: effect of transmitted phase and bubble size. IEEE Trans. Ultrason. Ferroelectr. Freq. Control 47, 1494-509.

Morita, T., Kurihara, H., Maemura, K., Yoshizumi, M., Yazaki, Y., 1993. Disruption of cytoskeletal structures mediates shear stress-induced endothelin-1 gene expression in cultured porcine aortic endothelial cells. J. Clin. Invest. 92, 1706-12.

Newman, C.M.H., Bettinger, T., 2007. Gene therapy progress and prospects: Ultrasound for gene transfer. Gene Ther 14, 465-475.

Ohl, C.-D., Arora, M., Ikink, R., de Jong, N., Versluis, M., Delius, M., Lohse, D., 2006. Sonoporation from jetting cavitation bubbles. Biophys. J. 91, 4285-95.

15 Park, J., Fan, Z., Deng, C.X., 2011. Effects of shear stress cultivation on cell membrane disruption and intracellular calcium concentration in sonoporation of endothelial cells. J. Biomech. 44, 164-9.

Pouliopoulos, A.N., Bonaccorsi, S., Choi, J.J., 2014. Exploiting flow to control the in vitro spatiotemporal distribution of microbubble-seeded acoustic cavitation activity 20 in ultrasound therapy. Phys. Med. Biol. 59, 6941-6957. 
Prentice, P., Cuschieri, A., Dholakia, K., Prausnitz, M., Campbell, P., 2005. Membrane disruption by optically controlled microbubble cavitation. Nat Phys 1, 107-110.

Qiu, Y., Luo, Y., Zhang, Y., Cui, W., Zhang, D., Wu, J., Zhang, J., Tu, J., 2010. The correlation between acoustic cavitation and sonoporation involved in ultrasoundmediated DNA transfection with polyethylenimine (PEI) in vitro. J. Control. Release $145,40-8$.

Qiu, Y., Zhang, C., Tu, J., Zhang, D., 2012. Microbubble-induced sonoporation involved in ultrasound-mediated DNA transfection in vitro at low acoustic pressures. J. Biomech. 45, 1339-45.

10 Radhakrishnan, K., Bader, K.B., Haworth, K.J., Kopechek, J. a, Raymond, J.L., Huang, S.-L., McPherson, D.D., Holland, C.K., 2013. Relationship between cavitation and loss of echogenicity from ultrasound contrast agents. Phys. Med. Biol. 58, 6541-63.

Rouleau, L., Farcas, M., Tardif, J.-C., Mongrain, R., Leask, R.L., 2010. Endothelial cell morphologic response to asymmetric stenosis hemodynamics: effects of spatial wall shear stress gradients. J. Biomech. Eng. 132, 081013.

Sato, M., Ohashi, T., 2005. Biorheological views of endothelial cell responses to mechanical stimuli. Biorheology 42, 421-441.

Schlicher, R.K., Radhakrishna, H., Tolentino, T.P., Apkarian, R.P., Zarnitsyn, V., Prausnitz, M.R., 2006. Mechanism of intracellular delivery by acoustic cavitation. 20 Ultrasound Med. Biol. 32, 915-24. 
Sennoga, C. a, Mahue, V., Loughran, J., Casey, J., Seddon, J.M., Tang, M., Eckersley, R.J., 2010. On sizing and counting of microbubbles using optical microscopy. Ultrasound Med. Biol. 36, 2093-6.

Stride, E.P., Coussios, C.C., 2010. Cavitation and contrast: the use of bubbles in ultrasound imaging and therapy. Proc. Inst. Mech. Eng. Part H J. Eng. Med. 224, $171-191$.

Tlaxca, J.L., Anderson, C.R., Klibanov, A.L., Lowrey, B., Hossack, J.A., Alexander, J.S., Lawrence, M.B., Rychak, J.J., 2010. Analysis of in vitro transfection by sonoporation using cationic and neutral microbubbles. Ultrasound Med. Biol. 36, 1907-18.

Unger, E.C., Porter, T., Culp, W., Labell, R., Matsunaga, T., Zutshi, R., 2004. Therapeutic applications of lipid-coated microbubbles. Adv. Drug Deliv. Rev. 56, $1291-314$.

Van Wamel, A., Kooiman, K., Harteveld, M., Emmer, M., ten Cate, F.J., Versluis, M., de Jong, N., 2006. Vibrating microbubbles poking individual cells: drug transfer into cells via sonoporation. J. Control. Release 112, 149-55.

Ward, M., Wu, J., Chiu, J.F., 1999. Ultrasound-induced cell lysis and sonoporation enhanced by contrast agents. J. Acoust. Soc. Am. 105, 2951-7.

Yu, H., Xu, L., 2014. Cell experimental studies on sonoporation: State of the art and remaining problems. J. Control. Release 174, 151-160. 
Zarins, C.K., Giddens, D.P., Bharadvaj, B.K., Sottiurai, V.S., Mabon, R.F., Glagov, S., 1983. Carotid bifurcation atherosclerosis. Quantitative correlation of plaque localization with flow velocity profiles and wall shear stress. Circ. Res. 53 , 502514.

5 Zeghimi, A., Uzbekov, R., Arbeille, B., Escoffre, J.-M., Bouakaz, A., 2012. Ultrastructural modifications of cell membranes and organelles induced by sonoporation. Ultrason. Symp. (IUS), 2012 IEEE Int.

Zhao, Y.-Z., Luo, Y.-K., Lu, C.-T., Xu, J.-F., Tang, J., Zhang, M., Zhang, Y., Liang, H.D., 2008. Phospholipids-based microbubbles sonoporation pore size and reseal of 10 cell membrane cultured in vitro. J. Drug Target. 16, 18-25.

Zhong, W., Chen, X., Jiang, P., Wan, J.M.F., Qin, P., Yu, A.C.H., 2013. Induction of endoplasmic reticulum stress by sonoporation: linkage to mitochondria-mediated apoptosis initiation. Ultrasound Med. Biol. 39, 2382-92.

Zhou, Y., Kumon, R.E., Cui, J., Deng, C.X., 2009. The size of sonoporation pores on the cell membrane. Ultrasound Med. Biol. 35, 1756-60.

Zhou, Y., Yang, K., Cui, J., Ye, J.Y., Deng, C.X., 2012. Controlled permeation of cell membrane by single bubble acoustic cavitation. J. Control. Release 157, 103-11. 


\section{FIGURE CAPTIONS}

Fig. 1. Ultrasound and flow chamber experimental setup. (a) A cell flow chamber was connected to a closed-loop flow system composed of a peristaltic pump, tubes, and

5 aspirator bottle. The bottle contained culture media, propidium iodide, and microbubbles. The cell chamber was submerged in degassed water at $37^{\circ} \mathrm{C}$. A single-element focused ultrasound transducer with a $0.5 \mathrm{MHz}$ center frequency was used for the treatment while a 7.5 MHz single-element passive cavitation detector was used to monitor acoustic cavitation activity. A PC-controlled waveform generator and a $50 \mathrm{~dB}$ power amplifier

10 drove the $0.5 \mathrm{MHz}$ FUS transducer while the captured cavitation emissions were filtered using a $1.25 \mathrm{MHz}$ high-pass filter, amplified through a $28 \mathrm{~dB}$ amplifier and then recorded with an oscilloscope. (b) Cell flow chamber. EA.hy926 cells were seeded in collagen treated flow chambers with a channel height of $400 \mu \mathrm{m}$ with a volume of $100 \mu$. The central chamber region was sonicated (solid red circle) while other regions (dotted green

15 circles) acted as non-sonicated control regions. Abbreviations: FUS: focused ultrasound; PCD: passive cavitation detector.

Fig. 2. Qualitative analysis of EA.hy926 cell viability and trans-membrane delivery due to ultrasound exposure using our initial parameters. (a) Prior to sonication, the cells

20 exhibited fluorescence (green) following administration of calcein-AM into the flow chamber. (b) Following the administration of PI (red) and microbubbles at a $1 \mathrm{X}$ clinical dose and sonication with a center frequency of $0.5 \mathrm{MHz}$, peak negative pressure of 110 $\mathrm{kPa}$, and 100-cycle pulse emitted at $0.2 \mathrm{~Hz}, 50$ times, cells were observed to dislodge from the flow chamber. (c) By decreasing the pressure to $75 \mathrm{kPa}$ and reducing the pulse 
length to 50 cycles we avoided dislodging of cells and observed a cell that exhibited trans-membrane delivery (red) while maintaining cell viability (green).

Fig. 3. Qualitative analysis of the EA.hy926 cell viability and trans-membrane delivery

5 for the controls and our final ultrasound parameters. In all experiments, calcein-AM was first administered into the chamber followed by the flowing of culture media and PI over cells seeded in a flow chamber. (a) Control cells without microbubbles (-MB) and without sonication (-FUS). (b) Control cells without microbubbles $(-\mathrm{MB})$, which were sonicated (+FUS) at our final parameters ( $f_{c}: 0.5 \mathrm{MHz}, \mathrm{P}_{\text {neg }}: 40 \mathrm{kPa}, \mathrm{PL}: 50$ cycles, PRF:

$\left.100.2 \mathrm{~Hz}, \mathrm{~N}_{\mathrm{p}}: 500\right)$. (c) Control cells with microbubbles (+MB) at a $50 \mathrm{X}$ clinical dose and without sonication (-FUS). (d) - (f) Cells with microbubbles (+MB) at a 50X clinical dose and sonication (+FUS) (f $\mathrm{f}_{\mathrm{c}}: 0.5 \mathrm{MHz}, \mathrm{P}_{\mathrm{pk}-\mathrm{neg}}: 40 \mathrm{kPa}$, PL: 50 cycles, PRF: $0.2 \mathrm{~Hz}, \mathrm{~N}_{\mathrm{p}}$ : 500), located at the upper-left edge (d), the center of the focal region (e) and the lowerright edge (f). Green and red cells indicate the presence of calcein-AM and PI, 15 respectively. Calcein-AM represents cell viability while PI represents trans-membrane delivery.

Fig. 4. Post-sonoporation fluorescence quantification for cell types A, B and C. (a) Examples of the different cell types within the sonicated region. (b) Lateral fluorescence 20 intensity profiles across the different cell types (calcein-AM: green line, PI: red line). (c) Average fluorescence intensity measured from an ensemble of 30 randomly selected cells of each type. Measurements are presented as mean \pm standard deviation $(n=30)$. $*: p<0.001$. 
Fig. 5. Acoustic cavitation characterization. During sonication of microbubbles flowing through the cell-seeded chamber, acoustic emissions were captured using a singleelement 7.5 MHz passive cavitation detector through a (a), (b) $5 \mathrm{MHz}$ or (c), (d) 1.25

$5 \mathrm{MHz}$ high-pass filter. Sonication parameters were set to the following: (a), (b) $\mathrm{f}_{\mathrm{c}}: 0.5$ MHz, $P_{\text {pk-neg: }}: 110 \mathrm{kPa}$, PL: 100 cycles, PRF: $0.2 \mathrm{~Hz}$, and $\mathrm{N}_{\mathrm{p}}:$ 50; and (c), (d) f $\mathrm{f}_{\mathrm{c}}: 0.5 \mathrm{MHz}$, $\mathrm{P}_{\mathrm{pk} \text {-neg: }}: 40 \mathrm{kPa}$, PL: 50 cycles, PRF: $0.2 \mathrm{~Hz}$, and $\mathrm{N}_{\mathrm{p}}$ : 500. (a), (c) The acoustic emissions energy was monitored throughout the sonication and was greater than the no microbubble control. Spectrograms of the microbubble acoustic emissions throughout the entire 10 sonication indicate the presence of (b) harmonic and broadband emissions or (d) harmonic emissions alone." 


\section{TABLE FORMATTING}

Table 1. Quantitative analysis of cell viability and trans-membrane delivery of the control and sonication experiments $\left(\mathrm{P}_{\mathrm{pk}-\mathrm{neg}}\right.$ : $40 \mathrm{kPa}$, PL: 50 cycles, PRF: $0.2 \mathrm{~Hz}$, and $\mathrm{N}_{\mathrm{p}}$ : 500,

$5 \mathrm{n}=2$ ). FUS: focused ultrasound exposure, MBs: flowing microbubbles at 50x concentration. Results are presented as mean \pm standard deviation.

\begin{tabular}{ccc}
\hline Condition & Cell viability (\%) & $\begin{array}{c}\text { Delivery rate to } \\
\text { viable cells }(\%)\end{array}$ \\
\hline Flow only & $99.77 \pm 0.11$ & 0 \\
Flow + FUS & $98.50 \pm 2.07$ & 0 \\
Flow + MBs & $98.70 \pm 1.26$ & 0 \\
Flow + FUS + MBs & $95.00 \pm 4.20$ & $62.6 \pm 4.3$ \\
\hline
\end{tabular}

